\title{
Lipid Core Abutting Lumen - Optical Coherence Tomography-Verified Thin-Cap Fibroatheroma Surrogate -
}

\author{
Ari Pollack, MD; Annapoorna S. Kini, MD; Jagat Narula, MD, PhD
}

$\mathbf{T}$ he ability of contemporary cardiovascular imaging techniques to identify patients at high risk for acute coronary syndrome (ACS) or those who may be at risk of peri-procedural myocardial infarction (MI) following percutaneous coronary intervention (PCI) has improved dramatically with the evolution of modalities such as intravascular ultrasound (IVUS), optical coherence tomography (OCT) and near-infrared spectroscopy. Imaging has facilitated our ability to inspect vascular wall pathology, plaque biology and composition while providing insight into atherosclerotic stability and evolution.

\section{Article p 808}

Fibrous cap thickness happens to be the most important determinant of plaque instability. ${ }^{1}$ OCT is a high-resolution intravascular imaging modality that measures the intensity of reflected light waves and converts these echoes into a highresolution tomographic image. By virtue of its high resolution $(10-15 \mu \mathrm{m})$, OCT is readily able to visualize and resolve the size and integrity of thin fibrous caps. ${ }^{2,3}$ And while the most important criterion related to vulnerable plaque detection may be the precise measurement of the fibrous cap itself, not all plaques that are thin, rupture; and perhaps other forces linked to plaque composition and transformation are at play. Absent cap thickness, extent of inflammation, and necrotic core size emerge as the determinants of vulnerability. A certainly impressive technology, the Achilles heel of OCT remains its low tissue penetration, thereby restricting its ability to quantitatively assess plaque area, morphology and vessel dimensions including remodeling. In contrast, IVUS is able to identify positive remodeling of the coronary arterial wall, higher lipid content and macrophage infiltration of plaques, which cannot be assessed using OCT.,5 The superior tissue penetration of IVUS occurs at the expense of poor axial resolution (100$150 \mu \mathrm{m})$, however, and limits the ability of IVUS to detect the presence of thin fibrous caps, the thickness of which ranges from 54 to $84 \mu \mathrm{m} .{ }^{1}$ Accordingly, the pursuit of the vulnerable plaque would ideally require a modality that allows for tissue penetration with exceptional resolution to enable comprehensive plaque analysis, architectural understanding of the vessel wall, and visualization of thin caps. IVUS has traditionally used the amplitude assessment of the radiofrequency wave backscatter for the gray-scale morphological characterization. The superimposition of spectral analysis in the frequency domain, which varies depending upon the underlying tissue characteristics, has allowed better definition of morphological components of plaque architecture. IVUS technology for plaque characterization is slowly evolving from the original $20-\mathrm{MHz}-$ phased array electronic systems to more recent high-frequency (40-45-MHz) mechanical probes that permit higher image quality. These strategies have been used in recent outcome studies of plaque characterization.

The PROSPECT study applied IVUS (virtual histology) for the assessment of plaque composition with the intention of identifying lesion characteristics portending increased future risk of ACS. ${ }^{6}$ In light of the poor resolution of IVUS, however, the presence of thin-cap fibroatheroma (TCFA) was defined as the absence of visible fibrous cap overlying a necrotic core. The study confirmed that the presence of IVUS-verified TCFA was a hallmark of future culprit lesion. In addition, the minimum luminal diameter and the magnitude of necrotic core were reported as the determinants of plaque instability. Other investigators have published several studies using integrated backscatter signal analysis for characterization of plaque composition. ${ }^{7}$

The morphological characteristics of the plaques that determine the risk of an acute event also determine the likelihood of peri-procedural myocardial injury following PCI. Studies have shown that peri-procedural MI is associated with worse cardiovascular outcome both in short- and long-term followup. ${ }^{8}$ The target lesions, in stable patients undergoing PCI, identified prior to revascularization as TCFA on OCT, indicate that the patients were at increased risk for the development of peri-procedural MI. ${ }^{9}$ In this issue of the Journal, Ozaki et al have used a high-frequency IVUS system $(40 \mathrm{MHz})$ with the purpose of achieving 2 goals: (1) to determine the feasibility of identifying TCFA with IVUS, as verified on OCT in the same sitting; and (2) to establish its association with the occurrence of peri-procedural MI following PCI. ${ }^{10}$ In a prospective, observational fashion, 100 consecutive patients with stable coronary artery disease undergoing PCI were evaluated on IVUS and OCT prior to intervention. Of these, 48 lesions had OCTconfirmed TCFA defined as cap thickness $<65 \mu \mathrm{m}$. After careful review of the IVUS data, the definition of IVUS TCFA was established as the collective presence of $>53.6 \%$ lipid plaque area, $\geq 1.03$ remodeling index, and the absence of any

The opinions expressed in this article are not necessarily those of the editors or of the Japanese Circulation Society.

Received February 23, 2015; accepted February 23, 2015; released online March 9, 2015

Icahn School of Medicine at Mount Sinai, New York, NY, USA

Mailing address: Jagat Narula, MD, PhD, Mount Sinai Medical Center, One Gustave L. Levy Place, Box 1030, New York, NY 10029,

USA. E-mail: narula@mountsinai.org

ISSN-1346-9843 doi:10.1253/circj.CJ-15-0211

All rights are reserved to the Japanese Circulation Society. For permissions, please e-mail: cj@j-circ.or.jp 
tissue intervening between lipid core and the lumen (termed as "lipid core abutting lumen", or LCAL). IVUS-defined TCFA demonstrated sensitivity, specificity, positive and negative predictive values of $73 \%, 90 \%, 88 \%$, and $78 \%$ using OCT-confirmed TCFA as the comparator. The investigators concluded that both IVUS-TCFA and OCT-TCFA were significant independent predictors of peri-procedural MI. The authors also concluded that patients with OCT-verified TCFA tended to have a larger plaque volume, higher remodeling index and longer lesion length as determined on gray-scale IVUS, and possessed more lipid plaque, wider lipid angle ( $>2$ quadrants), and less fibrous plaque. The advanced IVUS system used in that study possessed an axial resolution of at least $75 \mu \mathrm{m}$, which is of the same order of magnitude as the size of a thin fibrous cap segment. Thus, the authors were able to ascertain the presence of a thin fibrous cap by virtue of identifying LCAL plaque, and concluded that its presence was an independent predictor of OCT-confirmed TCFA.

Earlier investigators using IVUS have demonstrated the association of specific plaque characteristics, such as large plaque volume, with the development of post-procedural myocardial injury. ${ }^{11}$ OCT-confirmed TCFA has also been established as a risk factor for peri-procedural MI. ${ }^{9}$ In this study Ozaki et al extended the observations that TCFA confirmed on IVUS as well as OCT equally predicted myocardial injury following elective PCI, using both univariate and multivariate analysis. The relevance of peri-procedural injury should not be understated, as recently highlighted in a meta-analysis of 15 studies and 7,500 patients with normal baseline troponin level. ${ }^{8}$ Elevated peri-procedural troponin was associated with a significantly increased risk of in-hospital major adverse cardiovascular events (composite of all-cause death, MI, repeat target vessel PCI, and coronary artery bypass grafting [OR, 11.29; 95\% CI: $3.00-42.48$ ] or death [OR, 7.16; 95\% CI: 1.95-26.27]). Moreover, patients with PCI-related MI, as determined by the Joint European Society of Cardiology/American College of Cardiology Foundation/American Heart Association/World Health Federation Task Force, had a significantly increased risk of death (OR, 2.25; 95\% CI: 1.26-4.00) at 26-month follow-up. ${ }^{8}$ From a mechanistic standpoint, this event is likely to result from plaque disruption following stent implantation with resultant distal embolization from the site of intervention into both the epicardial and microvascular circulation. ${ }^{12}$ In support of this concept is the observation that increased fibrous plaque volume is not associated with myocardial injury. ${ }^{11}$ That said, the use of distal embolic protection devices, although valuable in the reduction of complications related to saphenous vein graft intervention, interestingly has not been shown to be effective in reducing peri-procedural MI in the native circulation. ${ }^{13}$

Ozaki et al appropriately allude to some limitations of their study including selection bias and the exclusion of calcified coronary disease that may raise concerns about wholesale clinical applicability. As most excellent investigations typically do, however, we are left with several questions to ponder. Do we possess the most complete invasive imaging technology in the form of advanced high-frequency IVUS? Is any further investigation even needed beyond OCT-TCFA in clinically significant lesions (wherein the extent of plaque and necrotic core volume may become moot)? As we inch closer to a better understanding of plaque biology, it will also be incumbent upon us to consider how treatment will change with evolution of diagnostic capability. Regardless of the unanswered questions, Ozaki et al deserve to be congratulated for a definitive study that establishes the lack of fibrous cap on IVUS as a surrogate for OCT-verified TCFA.

\section{Disclosures}

J.N. reports research support from Philips, GE and Panasonic Healthcare in the form of equipment grants to Mount Sinai. A.S.K. and A.P. report no conflicts of interest.

\section{References}

1. Narula J, Nakano M, Virmani R, Kolodgie FD, Petersen R, Newcomb $\mathrm{R}$, et al. Histopathologic characteristics of atherosclerotic coronary disease and implications of the findings for the invasive and noninvasive detection of vulnerable plaques. J Am Coll Cardiol 2013; 61: $1041-1051$.

2. Prati F, Regar E, Mintz GS, Arbustini E, Di Mario C, Jang IK, et al. Expert review document on methodology, terminology, and clinical applications of optical coherence tomography: Physical principles, methodology of image acquisition, and clinical application for assessment of coronary arteries and atherosclerosis. Eur Heart J 2010; 31: 401-415.

3. Yonetsu T, Bouma BE, Kato K, Fujimoto JG, Jang IK. Optical coherence tomography: 15 years in cardiology. Circ J 2013; 77: $1933-1940$.

4. Jang IK, Bouma BE, Kang DH, Park SJ, Park SW, Seung KB, et al. Visualization of coronary atherosclerotic plaques in patients using optical coherence tomography: Comparison with intravascular ultrasound. J Am Coll Cardiol 2002; 39: 604-609.

5. Varnava AM, Mills PG, Davies MJ. Relationship between coronary artery remodeling and plaque vulnerability. Circulation 2002; 105: 939-943.

6. Stone GW, Maehara A, Lansky AJ, de Bruyne B, Cristea E, Mintz GS, et al. A prospective natural-history study of coronary atherosclerosis. $N$ Engl J Med 2011; 364: 226-235.

7. Kawasaki M, Sano K, Okubo M, Yokoyama H, Ito Y, Murata I, et al. Volumetric quantitative analysis of tissue characteristics of coronary plaques after statin therapy using three-dimensional integrated backscatter intravascular ultrasound. J Am Coll Cardiol 2005; 45: 1946-1953.

8. Testa L, Van Gaal WJ, Biondi Zoccai GG, Agostoni P, Latini RA, Bedogni F, et al. Myocardial infarction after percutaneous coronary intervention: A meta-analysis of troponin elevation applying the new universal definition. QJM 2009; 102: 369-378.

9. Yonetsu T, Kakuta T, Lee T, Takahashi K, Yamamoto G, Iesaka Y, et al. Impact of plaque morphology on creatine kinase-MB elevation in patients with elective stent implantation. Int J Cardiol 2011; 146: $80-85$.

10. Ozaki Y, Ohota M, Ismail TF, Okumura M, Ishikawa M, Muramatsu T. Thin cap fibroatheroma defined as lipid core abutting lumen (LCAL) on integrated backscatter intravascular ultrasound: Comparison with optical coherence tomography and correlation with peri-procedural myocardial infarction. Circ J 2015; 79: 808-817.

11. Uetani T, Amano T, Ando H, Yokoi K, Arai K, Kato M, et al. The correlation between lipid volume in the target lesion, measured by integrated backscatter intravascular ultrasound, and post-procedural myocardial infarction in patients with elective stent implantation. Eur Heart J 2008; 29: 1714-1720.

12. Porto I, Selvanayagam JB, Van Gaal WJ, Prati F, Cheng A, Channon $\mathrm{K}$, et al. Plaque volume and occurrence and location of periprocedural myocardial necrosis after percutaneous coronary intervention: Insights from delayed-enhancement magnetic resonance imaging, thrombolysis in myocardial infarction myocardial perfusion grade analysis, and intravascular ultrasound. Circulation 2006; 114: 662-669.

13. Mauri L, Rogers C, Baim DS. Devices for distal protection during percutaneous coronary revascularization. Circulation 2006; 113: $2651-2656$. 